\title{
Small-angle neutron scattering studies of microenvironmental and structural changes of Pluronic micelles upon encapsulation of paclitaxel
}

\author{
Khalilalrahman Dehvari \\ Department of Chemical Engineering \& Materials Science/Environmental Technology Research \\ Centre, Yuan Ze University, Taoyuan City 320, Taiwan, R.O.C. \\ Email: Dehvarikh@gmail.com,Dehvari@saturn.yzu.edu.tw
}

Kuen-Song Lin *

Department of Chemical Engineering \& Materials Science/Environmental Technology Research Centre, Yuan Ze University, Taoyuan City 320, Taiwan, R.O.C.

*Corresponding author: kslin@ saturn.yzu.edu.tw

Tel: +886-3-4638800 ext. 2574, Fax:+886-3-4559373

\section{Boualem Hammouda}

Center for Neutron Research, National Institute of Standards and Technology, 100 Bureau Drive,

Stop 6102, Gaithersburg, Maryland 20899-6102

Email: boualem.hammouda@ nist.gov 


\begin{abstract}
Pluronic micelles represent a novel type of nanocarriers that enhance the therapeutic efficacy of paclitaxel (PTX) by increasing its solubility and circulation time. However, encapsulation or incorporation of a drug influences the micellar structure and subsequently alters the pharmacokinetic behavior of the drug delivery system. In this study, we investigated the structural properties of micelles after micellar solubilization of PTX. Proton nuclear magnetic resonance $\left({ }^{1} \mathrm{H}\right.$ NMR) and Fourier transform infrared spectroscopy (FTIR) studies showed that PTX was incorporated into the poly (propylene oxide) (PPO) blocks of the micelle cores. Smallangle neutron scattering (SANS) was used to probe changes in the core-shell structure of Pluronic P123 micelles as a function of increasing PTX and Pluronic concentration. Modelindependent analyses and cryogenic- transmission electron microscopy (cryo-TEM) confirmed that both pure and PTX-incorporated micelles were spherical. However, an increase in PTX concentration led to a slight increase in the micellar dimension and was associated with the appearance of the larger Pluronic-drug aggregates as confirmed by evaluation of cryo-TEM images. At higher Pluronic concentration and a constant PTX concentration, the dimensions of micelles increased, most likely due to the altered hydrophobic environment promoting the interaction of PPO and poly (ethylene oxide) (PEO) blocks.
\end{abstract}

Keywords: Pluronic prodrug, SANS, Paclitaxel, Nanocarrier, Drug delivery system. 


\section{Introduction}

Paclitaxel (PTX) is a well-known anti-cancer drug with proven efficacy against a variety of malignancies, including non-small cell lung, and metastatic breast, colon, and ovarian cancers [13]. However, PTX is highly hydrophobic with a very low water solubility $\left(0.3 \mu \mathrm{g} \mathrm{mL}^{-1}\right)$ that leads to precipitation of the drug upon dilution [4]. One strategy to overcome low solubility is modification of the drug to a prodrug form through the introduction of hydrophilic moieties with little or no pharmacological activity [5]. Prodrugs prolong drug circulation and enhance pharmacokinetics until they reach the target organ and then undergo an enzymatic and/or chemical transformation to release the active parent drug [6,7]. Consequently, dosage frequency can be reduced. Among the reported macromolecular PTX prodrugs, the implementation of Pluronic micelles as non-toxic vehicles for PTX delivery has been the focus of extensive studies $[1,2,8,9]$. The main advantages of Pluronics are P-glycoprotein mediated efflux inhibition [10], overcoming multidrug resistance (MDR) [11], and improvement of the enhanced permeability and retention (EPR) effect $[12,13]$. However, the pharmacokinetic behavior and accumulation of micelles in the tumor site via the EPR effect rely on the morphology and dimensions of the PTXloaded micelles.

Pluronic micelles can physically encapsulate and deliver drug nanocrystals to a tumor site, potentially preserving the original drug activity. However, this method suffers from low capacity and rapid release of the encapsulated drugs [14]. Indeed, dilution in bodily fluids is the main constraint for the physical encapsulation approach, which can lead to dissociation of micelles into monomer and drug precipitation. Alternatively, PTX molecules can be accommodated into the PPO hydrophobic core, while the tendency of PEO to dissolve in water enhances the solubility of the nanocarrier in the body fluid [1,15]. Nevertheless, incorporation of PTX into micelles also alters the balance of hydrophobic and hydrophilic domains in favor of PPO 
hydrophobicity, which in turn influences the structural properties of the micelles such as size, aggregation number, and dissolution [16]. Several studies suggested that PTX-pluronic conjugates results in increased drug solubility upon dilution $[1,8]$. However, conjugation may alter the biological properties of the drug, causing further complication in the characterization and regulatory approval [14].

In Pluronics, the micellar formation is highly sensitive to solvent composition and the presence of hydrophilic or hydrophobic molecules either interrupts or enhances the Pluronic selfassembly in solution $[17,18]$. On this basis, small-angle neutron scattering (SANS) is a useful tool to measure changes in the core-shell structural parameters such as micelle core and corona sizes, polydispersity, and aggregation number. It is the technique of choice to probe bulk of the system, providing quantitative data and real statistical averages. One further advantage is that the distinction between hydrogen and deuterium scattering, which helps to extract the structural information of a sample having similar X-ray scattering length density as solvent. Likewise, SANS has been shown to be an excellent tool in identifying hydrophobic domains in micellar systems composed of PEO-PPO-PEO triblock copolymers.

Pluronic-drug interactions have been the focus of many recent SANS investigations $[16,19,20]$. Sharma et al. reported that the presence of PTX decreased the Pluronic CMC by $40 \%$ while keeping the same core and corona size. The greatest changes were observed in the aggregation number and intermicellar interaction and inter-distance [16,19]. The same behavior was reported for the effect of ibuprofen on Pluronics P104 and P105, which each contain a similar number of PPO units but a different number of PEO units [20]. For both these Pluronics, larger PPO cores were observed as a result of drug incorporation. In another study, the encapsulation of flurbiprofen in solutions of Pluronics P123 and P103 was investigated by Alexander et al. [21] who demonstrated that incorporation of the drug into Pluronic building 
blocks reduced the CMC and led to an increased fraction of the micellized polymer as well as an increase in micelle dimension.

Therefore, the objective of the present work was to study the structural changes in PluronicPTX suspensions prepared by micellar solubilization and the encapsulation of PTX using P123 micelles. Firstly, the interactions of the drug with Pluronic blocks and micellar microenvironment were studied through proton nuclear magnetic resonance $\left({ }^{1} \mathrm{H} \mathrm{NMR}\right)$ and Fourier transforms infrared (FTIR) spectroscopies. Furthermore, the effects of drug and Pluronic concentration on micellar structures were studied using SANS and cryogenic transmission electron microscopy (cryo-TEM) techniques.

\section{Experimental Section}

\subsection{Materials}

All compounds including PTX, heavy water $\left(\mathrm{D}_{2} \mathrm{O}\right)$, acetonitrile, and Pluronic P123 (P123) were supplied by Sigma-Aldrich and used as received.

\subsection{Sample preparation}

Pluronic solutions were prepared by dissolving of the polymer in $\mathrm{D}_{2} \mathrm{O}$. The PTX-loaded P123 nanocarriers were prepared by an emulsion/solvent evaporation technique [22]. Briefly, the Pluronic and PTX in various feed volume ratios were dissolved in acetonitrile $(5 \mathrm{~mL})$, ultrasonicated for $20 \mathrm{~min}$ at room temperature and emulsified for $2 \mathrm{~h}$ in of PBS (10 mL). The solution was then evaporated under a stream of nitrogen. The resultant thin film was rehydrated at $70^{\circ} \mathrm{C}$ with $\mathrm{D}_{2} \mathrm{O}$ and ultrasonicated for $30 \mathrm{~min}$. The concentrations of Pluronic and PTX within the samples are presented in Table 1. The physical mixture of P123 and PTX prepared by 
recrystallization of PTX using acetonitrile followed by dissolution of the crystals in P123 solution. The incorporation and interaction of PTX were assessed by ${ }^{1} \mathrm{H}$ NMR and FTIR studies.

\section{Table 1}

Characteristics of the prepared samples.

\begin{tabular}{lccc}
\hline Sample name & P123/PTX & P123 volume & PTX volume \\
& ratio & fraction $\left(\times 10^{-3}\right)$ & fraction $\left(\times 10^{-5}\right)$ \\
\hline P123 1\% & - & 9.73 & - \\
P123 2\% & - & 19.3 & - \\
P 0.0015 & 667 & 9.73 & 1.07 \\
P 0.005 & 330 & 9.73 & 3.56 \\
P 0.01 & 200 & 19.3 & 14.1 \\
P2 0.005 & 200 & 37.8 & 13.8 \\
P 0.003 & 100 & 60.9 & 13.5 \\
P 0.03 & 33 & 9.73 & 21.4 \\
\hline
\end{tabular}

Note: "-" denotes "not available".

The ${ }^{1} \mathrm{H}$ NMR studies were conducted on a Bruker instrument operating at $500 \mathrm{MHz}$. The spectra of Pluronic and Pluronic-PTX solutions in $\mathrm{D}_{2} \mathrm{O}$ were measured at $25^{\circ} \mathrm{C}$. The samples were allowed to equilibrate at $25^{\circ} \mathrm{C}$ for at least 15 min prior to measurement. Infrared spectra were recorded on a Digilab FTIR spectrometer (ITS-40) with fully computerized data storage and data handling capability. For all the reported spectra, 64-scan data accumulation was used at a resolution of $4 \mathrm{~cm}^{-1}$. In order to observe small changes in band intensity and frequency, subtraction and baseline correction procedures were used. High-performance liquid chromatography (HPLC, Perkin Elmer Series 200) was used to measure the PTX concentrations. 
The drug loading efficiency was calculated from the following formulation: ((concentration of the feeding PTX-concentration of the free PTX) / concentration of the feeding PTX) $\times 100 \%$ ). The hydrodynamic sizes and zeta potential of the Pluronic micelles were determined using dynamic light scattering DLS on a Beckman Coulter ${ }^{\circledR}$ N4 PLUS machine at a wavelength of 514 $\mathrm{nm}$ and detection angle of $90^{\circ}$.

\subsection{Small angle neutron scattering experiments}

SANS measurements were carried out on the NGB30 SANS instrument at the National Institute of Standards and Technology (NIST) Center for Neutron Research, Gaithersburg, MD. Three configurations were used: one, with the detector offset by $25 \mathrm{~cm}$ and a sample-detector distance of $1 \mathrm{~m}$; two, with a sample-to-detector distance of $4 \mathrm{~m}$ and five inserted neutron guides to enhance flux on the sample and therefore improve statistics; three with the detector offset by $10 \mathrm{~cm}$ and a sample-detector distance of $13 \mathrm{~m}$ was applied to give a scattering vector range of 0.005-0.600 $\AA^{-1}$. Cold neutrons with a wavelength of $6.0 \AA$ were used, and all samples were measured in demountable titanium cells with $1 \mathrm{~mm}$ path length. Unless otherwise stated, samples were measured at $37^{\circ} \mathrm{C}$. The raw data were reduced using Igor Pro macros [23]. A standard data reduction method was used to correct for empty cell and blocked beam scattering and to rescale the radially averaged data to an absolute cross section scale. The reduced data were fitted using the core-shell model with constant poly core-shell ratio (PolyCoreShellRatio) of the NCNR Igor Pro data analysis package [24]. The value for solvent scattering length density (SLD) for $\mathrm{D}_{2} \mathrm{O}$ was kept constant at $6.38 \times 10^{-6} \AA^{-2}$ for all fittings.

The software program GNOM from ATSAS was also used to obtain information on the particle distance distribution function (PDDF), also known as the $P(r)$ function [25]. As an indirect transformational program for small-angle scattering data processing, GNOM reads in 
one-dimensional scattering curves and evaluates the PDDF. It provides a histogram of all distances between point pairs within particles weighted by the excess SLD at the point, which is useful to compute the radius of gyration $\left(R_{g}\right)$, maximum particle dimension $\left(D_{\max }\right)$, and Porod volume [26,27].

\subsection{Cryogenic-transmission electron microscopy}

The micellar structure in solution was visualized by cryo-TEM images. Specimens for cryoTEM were prepared with an FEI Vitrobot Mark IV vitrification system in a controlled environment. Samples were applied onto holey carbon films. After the blotting process, the grids were immediately plunged into liquid ethane and then stored in liquid nitrogen before imaging. Finally, TEM imaging was conducted on an FEI Tecnai T12 transmission electron microscope operating at $120 \mathrm{kV}$.

\section{Results and Discussion}

\subsection{Pluronic P123 micelles}

The solutions of P123 in $\mathrm{D}_{2} \mathrm{O}$ were studied as a function of both Pluronic and PTX concentrations. The structure of the $\mathrm{P} 123 \mathrm{PEO}_{20}-\mathrm{PPO}_{70}-\mathrm{PEO}_{20}$ block copolymer (Fig. 1) contains approximately $70 \%$ of hydrophobic PPO block with a relatively low CMC of $0.01 \mathrm{wt} / \mathrm{v} \%$ $[13,28,29]$. As a consequence, the starting concentration of $1 \mathrm{wt} / \mathrm{v} \%$ was well in excess of the CMC and thermodynamically stable micelles were observed for all the solutions. Above the CMC, micellization occurs via an endothermic process with entropic gains, in which hydrophobic PPO chains cluster themselves as a core surrounded by swollen hydrophilic PEO chains $[9,30]$. Likewise, the SANS data obtained for 1 and $2 \mathrm{wt} / \mathrm{v} \%$ solutions of P123 showed characteristic features of core-shell structure (Figs 2(a) and 2(c)). 

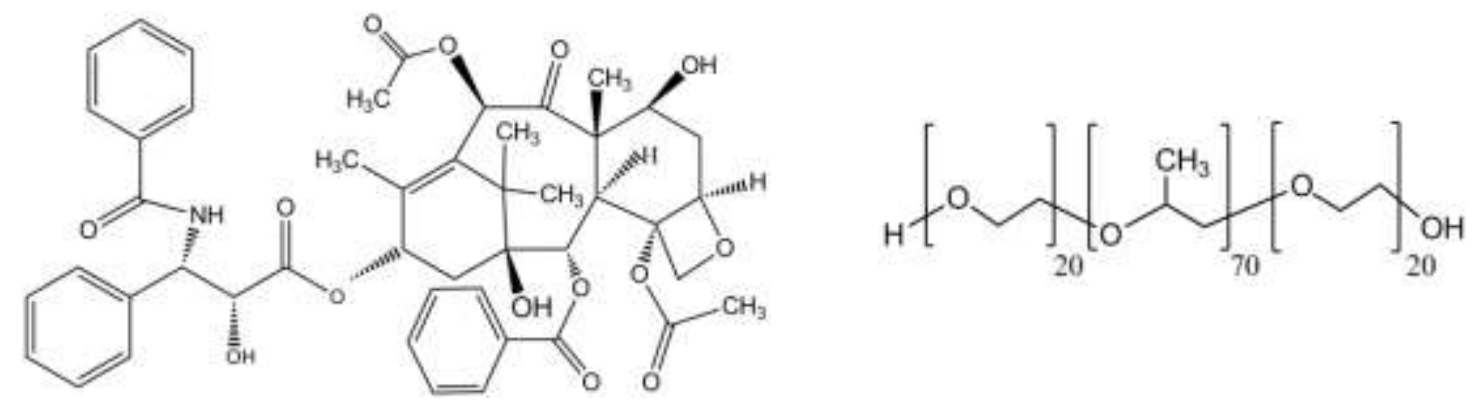

Fig. 1. The molecular structure of PTX (left) and Pluronic P123 (right).

To elucidate shape, the curve was Fourier-transformed into the PDFF in a model-free approach. As shown in Figs 2(b) and 2(d), the micelles are likely spherical. The results agree well with cryo-TEM microphotographs in the inset of Figs 2(a) and 2(c). From Table 2, it is evident that the difference between the neutron scattering length density of Pluronic blocks and $\mathrm{D}_{2} \mathrm{O}$ is sufficient to probe changes in the micellar structure of nanocarriers. Accordingly, the SANS data were fitted to the PolyCoreShellRatio model. As listed in Table 3, the core radius and corona thickness of P123 1\% obtained from the fitting results were $35 \AA$ and $34 \AA$, respectively. When the P123 concentration doubled, smaller micelles were observed. Such a trend was observed in the measurements of the hydrodynamic radius of P123 micelles with the DLS technique, in which a decrease in micellar size was observed in line with an increase of the Pluronic concentration [17]. Furthermore, the occurrence of oscillations in the P123 2\% PDDF curve suggests intermicellar interaction at this concentration [31]. This observation along with the higher population of micelles, which was indicated by higher number density of micelles in cryo-TEM image (Fig. 2(c), inset) led to a scattering intensity four times larger for P123 $2 \%$. 

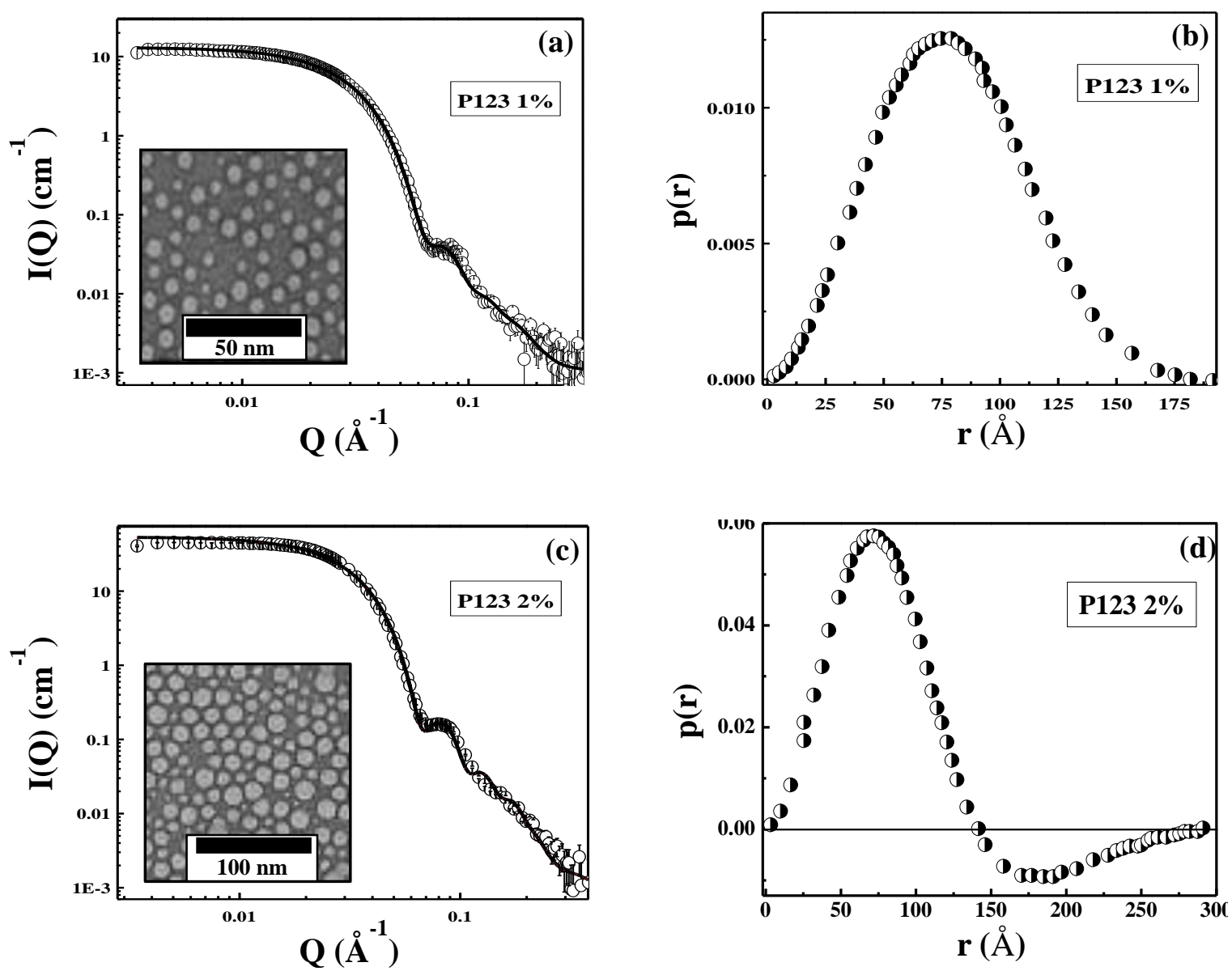

Fig. 2. ((a) and (c)) SANS with fitting curves along with cryo-TEM images (insets). ((b) and (d)) Pair distance distribution functions (PDDF) of Pluronic $\mathrm{P} 123$ in $\mathrm{D}_{2} \mathrm{O}$.

\section{Table 2}

Neutron scattering contrast of Pluronic blocks and PTX in $\mathrm{D}_{2} \mathrm{O}$.

\begin{tabular}{lcccc}
\hline Compound & Chemical & Density & Scattering length & Neutron contrast relative \\
& formula & $\left(\mathrm{g} / \mathrm{cm}^{3}\right)$ & density $\times 10^{-6}\left(\AA^{-2}\right)$ & to $\mathrm{D}_{2} \mathrm{O} \Delta \rho \times 10^{-6}\left(\AA^{-2}\right)$ \\
\hline $\mathrm{D}_{2} \mathrm{O}$ & $\mathrm{D}_{2} \mathrm{O}$ & 1.110 & 6.38 & - \\
$\mathrm{PEO}$ & $\mathrm{C}_{2} \mathrm{H}_{4} \mathrm{O}$ & 1.127 & 0.64 & 5.78 \\
$\mathrm{PPO}$ & $\mathrm{CH}_{3} \mathrm{CHCH}_{2} \mathrm{O}$ & 1.004 & 0.34 & 5.58 \\
Paclitaxel & $\mathrm{C}_{47} \mathrm{H}_{51} \mathrm{NO}_{14}$ & 1.390 & 2.08 & 4.30 \\
\hline
\end{tabular}




\section{Table 3}

SANS fitting results for Pluronic P123 micelles core-shell structure with increasing PTX and P123 concentration at $37^{\circ} \mathrm{C}$ using PolyCoreShellRatio model.

\begin{tabular}{lccccc}
\hline & $\begin{array}{c}\text { Core radius } \\
( \pm 0.6, \AA)\end{array}$ & $\begin{array}{c}\text { Shell thickness } \\
( \pm 0.1, \AA)\end{array}$ & $\begin{array}{c}\text { Polydispersity } \\
( \pm 0.002)\end{array}$ & $\begin{array}{c}\text { Core SLD } \\
\times 10^{-6} \\
\left( \pm 0.002, \AA^{-2}\right)\end{array}$ & $\begin{array}{c}\text { Shell SLD } \\
\times 10^{-6} \\
\left( \pm 0.003, \AA^{-2}\right)\end{array}$ \\
\hline P123 1\% & 35 & 34 & 0.13 & 2.88 & 3.77 \\
P123 2\% & 34 & 33 & 0.10 & 0.97 & 2.35 \\
P 0.0015 & 37 & 32 & 0.12 & 1.94 & 3.06 \\
P1 0.005 & 36 & 33 & 0.12 & 1.95 & 3.06 \\
P 0.03 & 34 & 35 & 0.12 & 1.76 & 2.93 \\
P 0.01 & 34 & 34 & 0.10 & 1.67 & 2.87 \\
P2 0.005 & 36 & 32 & 0.11 & 3.72 & 4.35 \\
P 0.003 & 37 & 33 & 0.12 & 3.76 & 4.42 \\
\hline
\end{tabular}

\subsection{Investigation of PTX and Pluronic interaction using FTIR and ${ }^{1} H$ NMR}

In order to study the underlying mechanism of PTX encapsulation, the interactions between PTX and P123 were examined through comparison of data from FTIR, ${ }^{1} \mathrm{H}$ NMR, SANS, HPLC, and cryo-TEM analyses. Fig. 2 represents the FTIR spectra of the P123 solution with different concentrations of PTX. The peak between $1000 \mathrm{~cm}^{-1}$ and $1100 \mathrm{~cm}^{-1}$ is assigned to the $\mathrm{C}-\mathrm{O}-\mathrm{C}$ stretch of the ether bond, which is a feature of the Pluronic copolymer [32]. This bond along with other distinctive peaks related to PEO structure faded out at low concentration of the pluronic, resulting in single broad peak around $1100 \mathrm{~cm}^{-1}$. As can be seen in Fig. 1, the structure of PTX offers a variety of functional groups to form bonds with P123. FTIR spectra of the samples loaded with PTX showed additional peaks of PTX molecule at 1,072, 1,248, 1,370, and 2,965 
$\mathrm{cm}^{-1}$ that were assigned to $\mathrm{C}-\mathrm{O}$ stretch, $\mathrm{C}-\mathrm{N}$ stretch, $\mathrm{CH}_{3}$ bending, and $=\mathrm{C}-\mathrm{H}$ structure, respectively $[33,34]$. However, resonance intensities and morphologies varied between samples. For a constant Pluronic concentration, the addition of an increased concentration of PTX did not affect the bands attributed to the PEO structure. As the Pluronic contents went up, a majority of PTX signals vanished, indicating that successful incorporation of PTX into micelles was clearly dependent on both PTX and Pluronic concentrations.

Fig. 4 shows the locally expanded ${ }^{1} \mathrm{H}$ NMR spectra of the $\mathrm{EO}-\mathrm{CH}_{2}-$, $\mathrm{PO}-\mathrm{CH}_{2}-$, and $\mathrm{PO}-$ $\mathrm{CH}_{3}$ regions of $\mathrm{P} 123$ in $\mathrm{D}_{2} \mathrm{O}$ [35]. The spectrum of the Pluronic solution showed a sharp singlet peak for the EO $-\mathrm{CH}_{2}-$ protons (Fig. 4(a)) with a slightly linear $\sim 0.01 \mathrm{ppm}$ upfield shift after addition of the drug. This observation indicates that the local environment of the PEO blocks was almost unchanged, with the PEO blocks still in contact with the aqueous solvent.

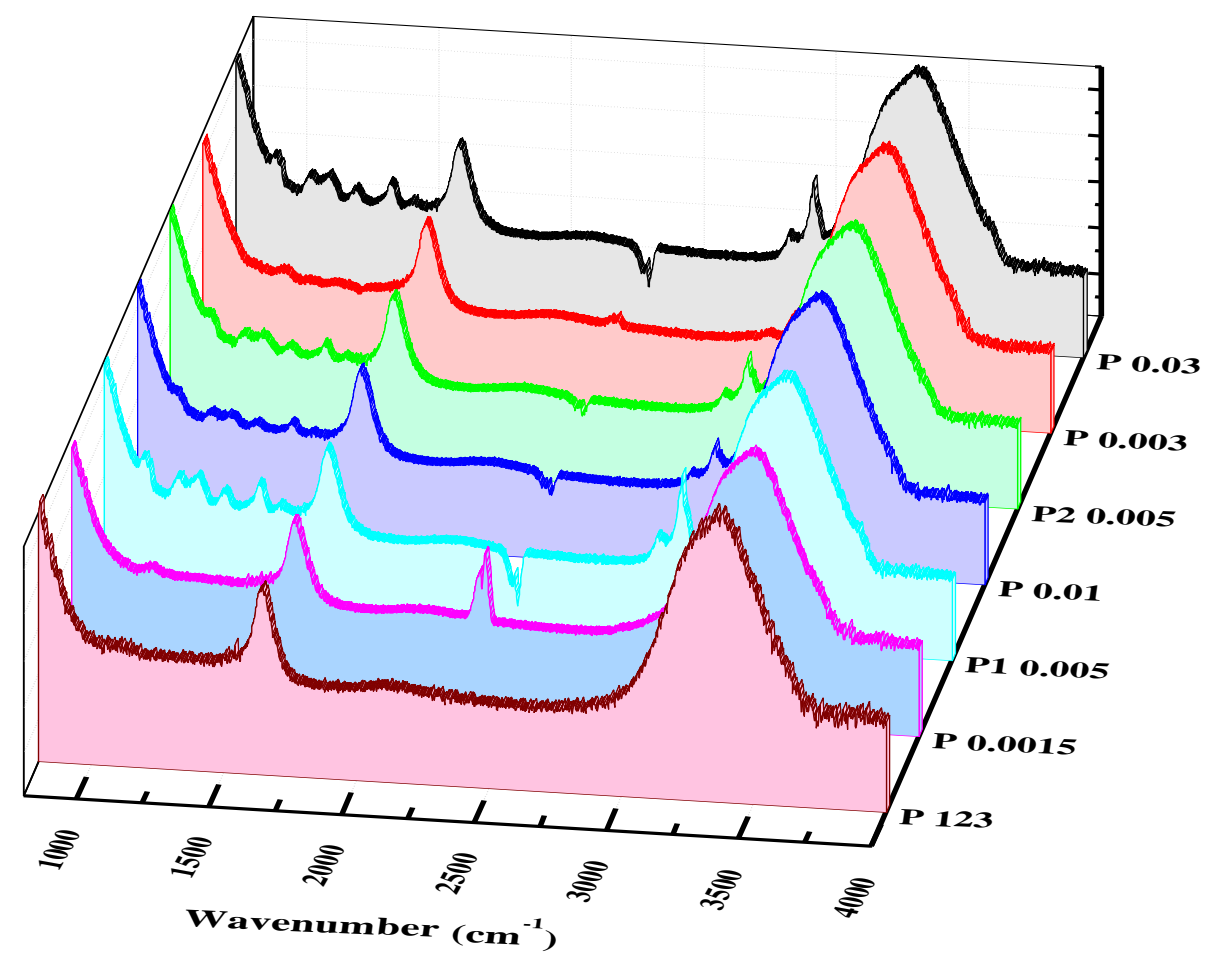

Fig. 3. FTIR spectra of Pluronic P123 before and after encapsulation of different concentrations of PTX. 
In contrast, $\mathrm{PO}-\mathrm{CH}_{2}-$ (Fig. 4(b)) and $\mathrm{PO}-\mathrm{CH}_{3}$ (Fig. 4(c)) regions experience significant changes in line shape and width, as well as chemical shift. As a hydrophobic drug, PTX interactes with the $\mathrm{PO}-\mathrm{CH}_{2}-/-\mathrm{CH}_{3}$ protons and affectes the hydrophobic microenvironment formed by PO blocks in the micelle core. The resonance at 3.4 was considered as one of the non-equivalent PO $-\mathrm{CH}_{2}-$ protons, specifically the intramolecular $(\mathrm{C}-\mathrm{H}) \cdots \mathrm{O}$ hydrogen bond between the PO $-\mathrm{CH}_{2}-$ protons and the ether oxygen [36,37]. Some functional groups of PTX, such as N-H and $\mathrm{O}-\mathrm{H}$, can substitute or influence any side of this hydrogen bond. Therefore, substantial changes in the peak width and height as a function of PTX concentration arose from the formation of hydrogen bonds between PPO and PTX. Based on the FTIR and ${ }^{1} \mathrm{H}$ NMR measurement, it was clear that PTX was accommodated into the micelle core through interaction with PPO units.

\subsection{Effect of PTX on the structure of P123 micelles}

The effects of PTX on the structural properties of micelles were studied for the samples with varying Pluronic and PTX concentrations. As shown in Fig. 5 (a), at low Q, a slight increase in the scattering intensity was detected as a function of PTX concentration. In particular, samples with the highest initial PTX concentration and PTX/P123 ratio displayed a higher scattering intensity at low Q that indicated the formation of larger aggregates; this is a form of clustering (Fig. 5 (a), inset) [38]. Additionally, the upturn in low Q may suggest the presence of the second population of bigger nanoparticles. This feature became more pronounced for the samples with a higher PTX/P123 ratio, which may imply that the PTX crystals grow when excess quantities are available. A plot of $\mathrm{I}(\mathrm{Q})^{*} \mathrm{Q}^{2}$ vs. $\mathrm{Q}$, the so-called Kratky plot, was used to examine the rough changes in the shape of aggregates. As shown in Fig. 5(b), the Kratky plot exhibited a bellshaped peak at low Q for all the samples; indicating a globular shape. It is clear that addition of PTX did not cause a substantial change in aggregates foldness. 

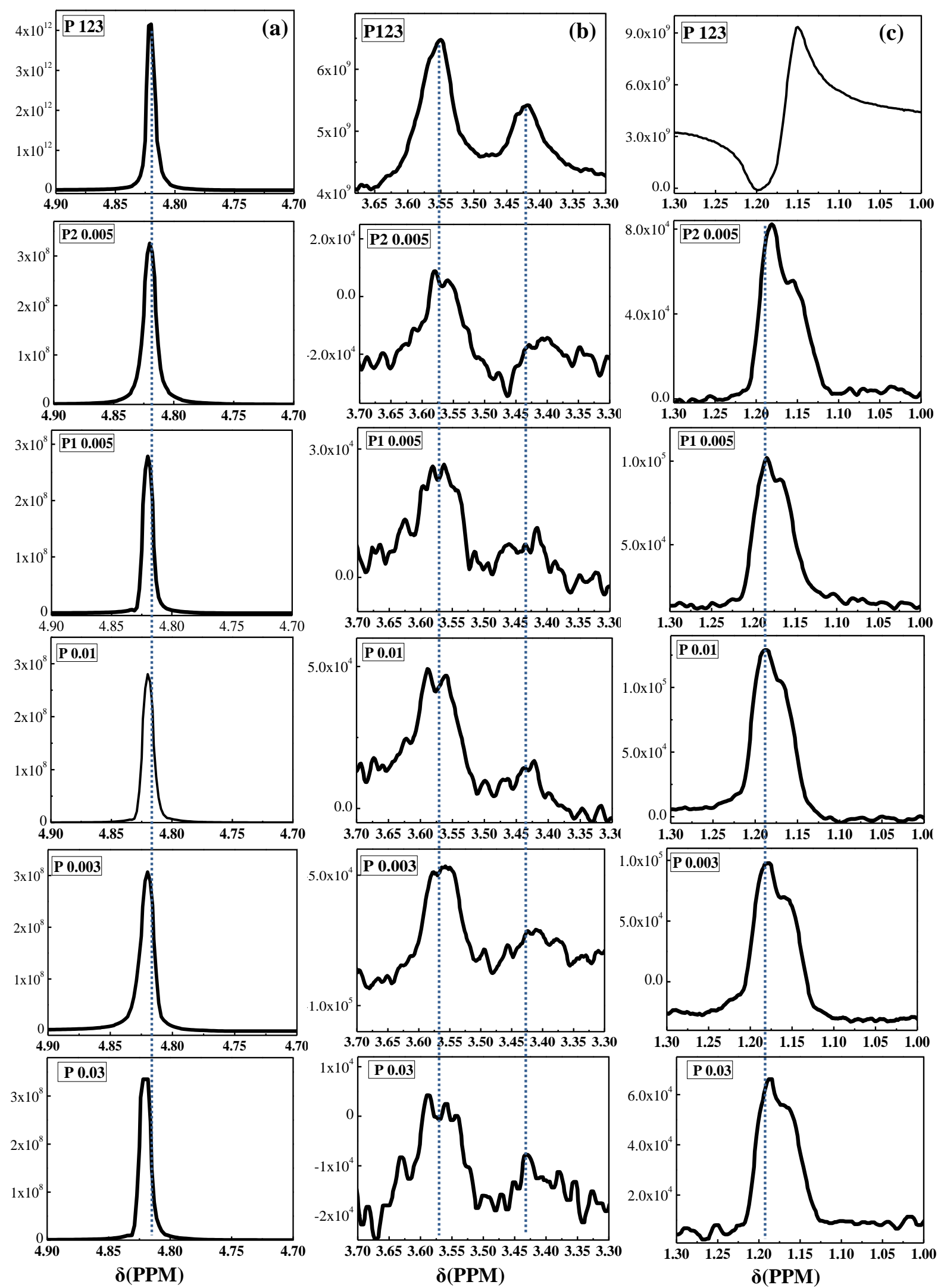

Fig. 4. ${ }^{1} \mathrm{H}$ NMR spectra of Pluronic $\mathrm{P} 123$ with various concentrations of $\mathrm{PTX}$ in $\mathrm{D}_{2} \mathrm{O}$ solution showing the following signals: (a) $\mathrm{CH}_{2}-\mathrm{O}$ in $\mathrm{EO}$, (b) $\mathrm{PO}-\mathrm{CH}_{2}-\mathrm{C}-\mathrm{CH}-$, and (c) $\mathrm{PO}-\mathrm{CH}_{3}$ 
Table 4 compares $\mathrm{R}_{\mathrm{g}}, \mathrm{D}_{\max }$, Porod volume, and the exponent between the samples. Two general trends can be seen in these results: firstly, the effect of Pluronic concentrations that led to lower $D_{\max }$ and $R_{g}$ values; secondly, the positive correlation of $D_{\max }$ with the higher PTX concentration at a constant Pluronic concentration of 1\%. The HPLC results in Fig. 6(a) demonstrated that the drug loading efficiency increased after addition of a higher PTX concentration. Thus bigger micelles were expected to see in response to a higher loading of the drug. Exponents more than four are indicative of either a diffuse interface [39], which would apply, for example, to a large PTX surface with an adsorbed Pluronic, or of increased intermicellar interactions. Furthermore, the presence of oscillations in the PDDF curves is a clear signature of intermicellar interaction [31].

\section{Table 4}

The maximum particle dimension $\left(\mathrm{D}_{\max }\right)$, radius of gyration $\left(\mathrm{R}_{\mathrm{g}}\right)$, Porod volume, and exponents derived from SANS data using PDDF function.

\begin{tabular}{lcccc}
\hline & $\mathrm{D}_{\max }( \pm 0.6, \AA)$ & $\mathrm{R}_{\mathrm{g}}( \pm 0.6, \AA)$ & Porod volume $\left(\AA^{3}\right)$ & Power $( \pm 0.1,--)$ \\
\hline P123 1\% & 195 & 60 & $1,355,890$ & 4.0 \\
P 0.0015 & 184 & 59 & $1,332,690$ & 4.0 \\
P1 0.005 & 232 & 56 & $1,268,870$ & 4.2 \\
P 0.03 & 195 & 59 & $1,315,620$ & 4.2 \\
P123 2\% & 302 & 29 & $1,097,500$ & 4.0 \\
P 0.01 & 332 & 29 & 997,707 & 4.0 \\
P2 0.005 & 257 & 40 & 118,8730 & 4.0 \\
P 0.003 & 253 & 55 & $1,286,850$ & 4.2 \\
\hline
\end{tabular}


It can be seen from Fig. 2 (b) that the PDDF curve of P123 2\% goes through a negative minimum and shows a strong oscillation that vanishes at very long distances. Likewise, the PDDF of the sample P0.01 (P123 2\% with PTX) in Fig. 6(b) also exhibited a similar feature. Although the $R_{g}$ and $D_{\max }$ values fluctuated between samples, as the Pluronic concentration went up they showed two opposing trends. The values of Rg declined in concentrated samples, but conversely, the overall trend for $\mathrm{D}_{\max }$ was upward. A closer examination of the results suggested that the variation of $R_{g}$ and $D_{\max }$ was attributed to the intermicellar interaction of the samples and the occurrence of large drug and P123 clusters.

\subsubsection{Characterization of core-shell structure of micelles}

The PolyCoreShellRatio model for sphere form factor was used to study the structure of the micelles in response to the addition of PTX. As shown in Table 3, addition of the drug was associated with a slight change in both core radius and shell thickness. Similar results were reported for PTX solubilization by Pluronic F127 [19] and PEtOz-PCL copolymers [40]. However, many studies demonstrated that Pluronic micelles increased in size as a result of incorporation of a hydrophobic drug [20,21,41-43]. As discussed in preceding sections, comparison of scattering intensities in the low Q region in Fig. 5(a) indicated that the addition of PTX favors clustering of the Pluronic micelles or PTX. Likewise, the comparison of sample zeta potentials (Table 5) indicated a higher tendency toward micellar aggregation upon addition of the

drug. In fact, due to the incorporation of hydrophobic entities in the PPO core, expansion of micellar dimensions was expected $[29,44,45]$. Although the core radius was slightly larger than that of pure Pluronic micelles, the trend was reversed at higher drug concentration. This might be 
explained by the thermodynamically favored crystallization of PTX as the solvent (acetonitrile) evaporated.
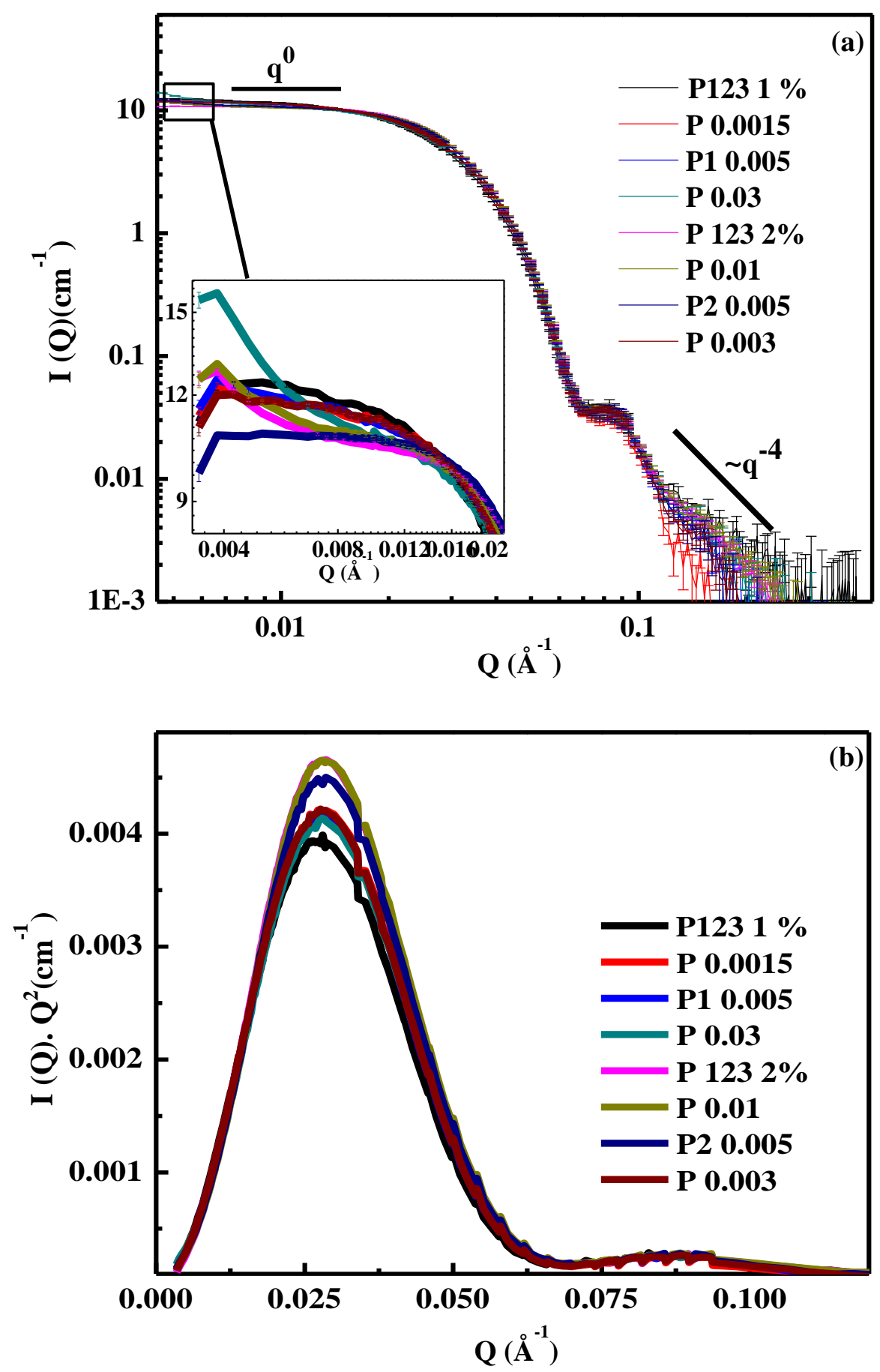
Fig. 5. (a) Small angle neutron scattering spectra of Pluronic P123 with increasing concentration of PTX in $\mathrm{D}_{2} \mathrm{O}$ at $37^{\circ} \mathrm{C}$ and (b) Kratky plots of SANS data.
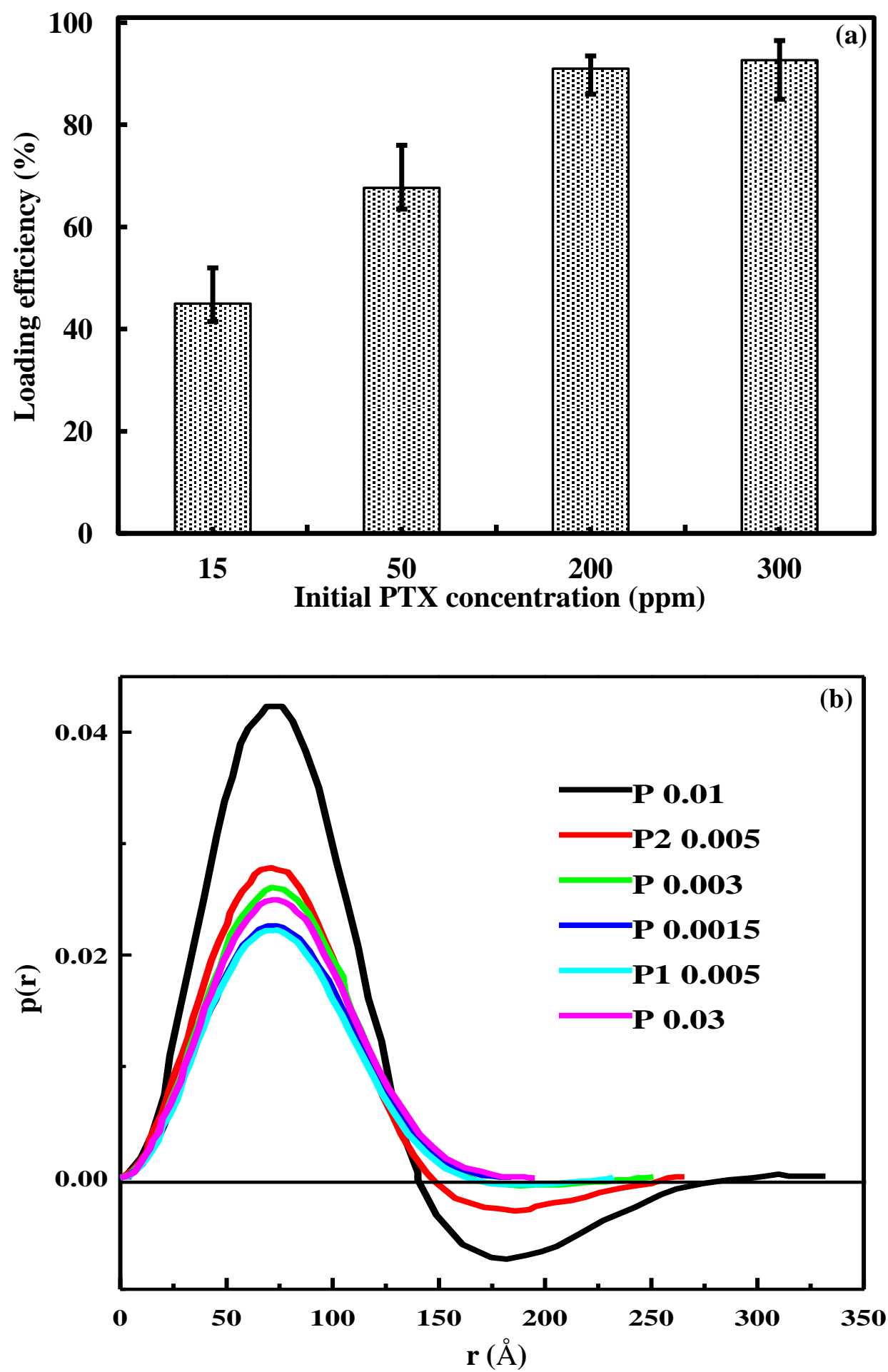
Fig. 6. (a) Loading efficiency of PTX into Pluronic micelles as a function of initial drug concentration. Statistical error bars correspond to one standard deviation. (b) Pair distance distribution function (PDDF) of Pluronic P123 with encapsulated PTX in $\mathrm{D}_{2} \mathrm{O}$. 


\section{Table 5}

Hydrodynamic size and zeta potential of the selected samples.

\begin{tabular}{lcc}
\hline Sample & Particle size $(\mathrm{nm})$ & Zeta potential $(\mathrm{mV})$ \\
\hline P123 $1 \%$ & 29 & -14.5 \\
P123 2\% & 25 & -17.5 \\
P 0.01 & 22 & 1.2 \\
P 0.003 & 36 & -13 \\
\hline
\end{tabular}

In this case, the crystals grow rapidly as a function of increased PTX concentration to form large particles. Generally, SANS and HPLC samples were centrifuged or filtered to remove large particles and achieve homogeneous solutions. Consequently, volume fractions of drug and Pluronic drop in the purified sample. In other words, the population of PTX particles is relatively much lower than that of Pluronic micelles and only can be seen as an upturn in the low Q region. This observation was in line with the increased $\mathrm{D}_{\max }$ observed for higher PTX concentrations.

The effect of Pluronic concentration on the core-shell structure of the micelles was investigated through comparison of three samples with constant PTX concentration and varying Pluronic concentration namely, P0.01, P2 0.005, and P0.003. In contrast to the pure Pluronic, in the presence of PTX, micellar size increased for higher Pluronic concentrations. It was hypothesized that the presence of the drug created a more hydrophobic microenvironment in the core, and the addition of new PPO blocks formed a compact structure with a higher aggregation number of this unit in the core. However, a larger the number of PPO units in the core reduced the influence of PTX on the Pluronic micelles. The new environment leads to greater interaction 
between PPO and PEO blocks, which was previously interrupted by the presence of PTX. Therefore, the solvent volume fraction in both the core and shell increased.

\subsubsection{Micellar structure visualization using cryo-TEM studies}

A Pluronic micellar formulation of PTX can increase the solubility of PTX and extend the elimination half-life, giving rise to improved antitumor efficacy and fewer side effects [46,47]. This goal can be achieved by either encapsulation of drug entities inside the micelles or by attachment of the monomers or micelles on the surface resulting in the suspension of the PTX nanocrystals. In order to visualize the micellar morphologies and validate the SANS results, cryo-TEM images were taken of PTX encapsulated samples. As depicted in Figs 7(a-d), the samples exhibited spherical micelles with different polydispersity and micellar number density. The cryo-TEM microphotos (Figs 7(e) and 7(f)) of the samples containing solubilized PTX particles without further filtration revealed the existence of complex core-shell structure (approximately 200-500 nm in size). The clusters consisted of PTX crystals coated or surrounded by a Pluronic shell or micelles and remained soluble in the aqueous solution. These samples exhibited a vague solution although no drug precipitation was observed. Also, no significant changes were seen in micellar sizes, which may explain the SANS fitting results indicating similarly sized micelles. Lin et al. reported similar results in a study on the adsorption behavior of Pluronic F127 onto carbon black particles dispersed in aqueous media [48].

Overall, the final structures of micelles vary with changing concentrations of PTX and Pluronic in solution. Because PTX incorporates into P123 through interaction with PPO, a higher concentration of Pluronic leads to increased PTX solubilization. 

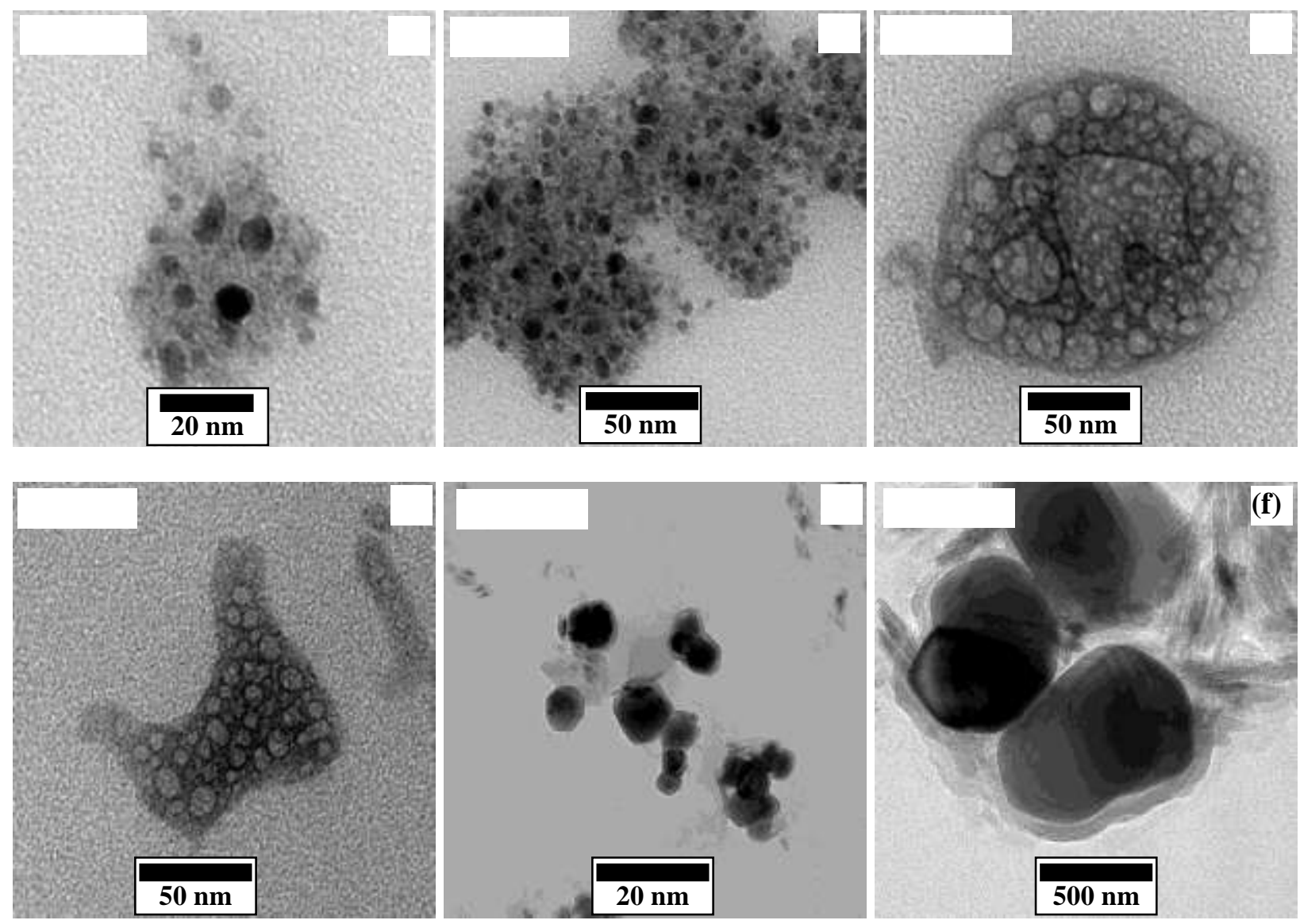

Fig. 7. ((a) - (d)) cryogenic-transmission electron microscopy images of selected Pluronic sample after encapsulation of PTX. (e) and (f) Cryo-TEM images taken from samples containing PTX crystal without further filtration or centrifugation.

However, an inevitable consequence of the increased Plutonic concentration is that higher drug incorporation is correlated to an increase in the number density of the micelle, and in turn affects gelation through the micellar volume fraction. Encapsulation of PTX nanocrystals was associated with the co-existence and dynamic equilibrium of pure and PTX-loaded P123 micelles as well as dispersed PTX nanoparticles in an aqueous solution. Therefore, proper matching of the Pluronic 
and PTX concentration leads to control over the mechanism of encapsulation and formulation of an efficient nanoparticle drug delivery entity.

\section{Conclusions}

The structural properties of Pluronic and PTX-loaded micelles, and the effects of varying drug and Pluronic P123 concentrations on the micellar structure have been studied. The results showed that higher PTX concentration led to higher scattering intensity in the low Q region, indicating that complex clusters of PTX and P123 emerged in the samples. Furthermore, SANS measurements showed that increase in P123 concentration with constant PTX concentration were correlated with the larger core dimensions and subtle changes in shell thickness. The following hypothesis has been put forward to explain these results. As the interaction of PTX with PPO blocks of Pluronic was confirmed by ${ }^{1} \mathrm{H}$ NMR and FTIR studies, encapsulation of PTX makes the core a highly hydrophobic compact environment, where PPO and PEO interactions are hindered. However, the addition of Pluronic into the solution moderated this environment and improved the block interaction, leading to an increased volume fraction of solvent in both core and shell. As Pluronics have attracted tremendous attention in the field of nanomedicine, it is necessary to study the underlying mechanism of drug encapsulation and the effects on the

micellar structure before carriers can be transferred to the clinical setting. Such studies will promote the development of sustainable stimuli-responsive nanocarriers for controlled-release drug delivery systems.

\section{Disclaimer/Acknowledgments}

The identification of commercial products does not imply endorsement by the National Institute of Standards and Technology (NIST) nor does it imply that these are the best for the 
purpose. This work is based upon activities supported in part by the National Science Foundation (NSF) under Agreement No. DMR 0944772.

\section{References}

[1] Du Z, Zhang Y, Xu H, Lang M. Functionalized pluronic-b-poly([varepsilon]-caprolactone) based nanocarriers of paclitaxel: Solubilization, antiproliferative efficacy and in vivo pharmaceutic kinetics. J Mater Chem B 2015;3:3685-94.

[2] Dehvari K, Chen Y, Tsai YH, Tseng SH, Lin KS. Superparamagnetic iron oxide nanorod carriers for paclitaxel delivery in the treatment and imaging of colon cancer in mice. J Biomed Nanotechnol 2016;12:1734-45.

[3] Kala S, Mak ASC, Liu X, Posocco P, Pricl S, Peng L, et al. Combination of dendrimernanovector-mediated small interfering RNA delivery to target Akt with the clinical anticancer drug paclitaxel for effective and potent anticancer activity in treating ovarian cancer. J Med Chem 2014;57:2634-42.

[4] Zhang H, Hu H, Zhang H, Dai W, Wang X, Wang X, et al. Effects of PEGylated paclitaxel nanocrystals on breast cancer and its lung metastasis. Nanoscale 2015;7:10790-800.

[5] Skwarczynski M, Hayashi Y, Kiso Y. Paclitaxel prodrugs: Toward smarter delivery of anticancer agents. J Med Chem 2006;49:7253-69.

[6] Liu T, Jia T, Yuan X, Liu C, Sun J, Ni Z, et al. Development of octreotide-conjugated polymeric prodrug of bufalin for targeted delivery to somatostatin receptor 2 overexpressing breast cancer in vitro and in vivo. Int J Nanomedicine 2016;11:2235—50.

[7] Rautio J, Kumpulainen H, Heimbach T, Oliyai R, Oh D, Jarvinen T, et al. Prodrugs: Design and clinical applications. Nat Rev Drug Discov 2008;7:255-70.

[8] Chen Y, Zhang W, Huang Y, Gao F, Sha X, Fang X. Pluronic-based functional polymeric mixed micelles for co-delivery of doxorubicin and paclitaxel to multidrug resistant tumor. Int $\mathrm{J}$ Pharm 2015;488:44-58.

[9] Emami J, Rezazadeh M, Hasanzadeh F, Sadeghi H, Mostafavi A, Minaiyan M, et al. Development and in vitro/in vivo evaluation of a novel targeted polymeric micelle for delivery of paclitaxel. Int J Biol Macromol 2015;80:29-40. 
[10] Wei Z, Yuan S, Hao J, Fang X. Mechanism of inhibition of P-glycoprotein mediated efflux by Pluronic P123/F127 block copolymers: Relationship between copolymer concentration and inhibitory activity. Eur J Pharm Biopharm 2013;83:266-74.

[11] Zhang W, Shi Y, Chen Y, Ye J, Sha X, Fang X. Multifunctional Pluronic P123/F127 mixed polymeric micelles loaded with paclitaxel for the treatment of multidrug resistant tumors. Biomaterials 2011;32:2894-906.

[12] Chen L, Sha X, Jiang X, Chen Y, Ren Q, Fang X. Pluronic P105/F127 mixed micelles for the delivery of docetaxel against taxol-resistant non-small cell lung cancer: Optimization and in vitro, in vivo evaluation. Int J Nanomedicine 2013;8:73-84.

[13]Liu Z, Wang Y, Zhang J, Li M, Liu Y, Zhang N. Pluronic P123-docetaxel conjugate micelles: Synthesis, characterization, and antitumor activity. J Biomed Nanotechnol 2013;9:2007-16.

[14] Rangel-Yagui CO, Junior AP, Tavares LC. Micellar solubilization of drugs. J Pharm Pharmaceut Sci 2005;8:147-63.

[15] Gupta R, Shea J, Scafe C, Shurlygina A, Rapoport N. Polymeric micelles and nanoemulsions as drug carriers: Therapeutic efficacy, toxicity, and drug resistance. J Control Release 2015;212:70-7.

[16] Sharma PK, Reilly MJ, Bhatia SK, Sakhitab N, Archambault JD, Bhatia SR. Effect of pharmaceuticals on thermoreversible gelation of PEO-PPO-PEO copolymers. Colloids Surf, B 2008;63:229-35.

[17] Ci T, Li T, Chen L, Chang G, Yu L, Ding J. Effects of "mature micelle" formation of Pluronic P123 on equilibrium between lactone and carboxylate forms of 10-hydrocamptothecin in water. Polym Chem 2013;4:3245-55.

[18] Sarkar B, Alexandridis P. Self-assembled block copolymer-nanoparticle hybrids: Interplay between enthalpy and entropy. Langmuir 2012;28:15975-86.

[19] Sharma PK, Reilly MJ, Jones DN, Robinson PM, Bhatia SR. The effect of pharmaceuticals on the nanoscale structure of PEO-PPO-PEO micelles. Colloids Surf, B 2008;61:53-60.

[20] Foster B, Cosgrove T, Hammouda B. Pluronic triblock copolymer systems and their interactions with ibuprofen. Langmuir 2009;25:6760-6.

[21] Alexander S, Cosgrove T, Prescott SW, Castle TC. Flurbiprofen encapsulation using pluronic triblock copolymers. Langmuir 2011;27:8054-60.

[22] Tao Y, Han J, Wang X, Dou H. Nano-formulation of paclitaxel by vitamin E succinate functionalized pluronic micelles for enhanced encapsulation, stability and cytotoxicity. Colloids Surf, B 2013;102:604-10. 
[23] Kline S. Reduction and analysis of SANS and USANS data using IGOR Pro. J Appl Crystallogr 2006;39:895-900.

[24] Hayter JB. Physics of amphiphiles--micelles, vesicles, and microemulsions. In: DeGiorgio V, Corti M, editors. Proceeding of the international school of physics; 1983. p. 59-93.

[25] Svergun D. Determination of the regularization parameter in indirect-transform methods using perceptual criteria. J Appl Crystallogr 1992;25:495-503.

[26] Jensen GV, Shi Q, Hernansanz MJ, Oliveira CL, Deen GR, Almdal K, et al. Structure of PEP-PEO block copolymer micelles: Exploiting the complementarity of small-angle X-ray scattering and static light scattering. J Appl Crystallogr 2011;44:473-82.

[27] Glatter O. New method for evaluation of small-angle scattering data. J Appl Crystallogr 1977;10:415-21.

[28] Dehvari K, Lin KS, Wang SS. Structural characterization and adsorption properties of Pluronic F127 onto iron oxides magnetic nanoparticles. J Nanosci Nanotechnol 2014;14:2361-7.

[29] Alexander S, de Vos WM, Castle TC, Cosgrove T, Prescott SW. Growth and shrinkage of Pluronic micelles by uptake and release of flurbiprofen: Variation of $\mathrm{pH}$. Langmuir 2012;28:6539-45.

[30] Dehvari K, Lin KS, Wang SS. Small angle X-ray scattering characterization of multifunctional iron oxide-Pluronic nanocarriers: Effect of temperature and drug encapsulation. Nanosci, Nanotech Let 2016;8:667-70.

[31] Hassan PA, Fritz G, Kaler EW. Small angle neutron scattering study of sodium dodecyl sulfate micellar growth driven by addition of a hydrotropic salt. J Colloid Interface Sci 2003;257:154-62.

[32] Hermida-Merino D, Newby GE, Hamley IW, Hayes W, Slark A. Microphase separation induced in the melt of pluronic copolymers by blending with a hydrogen bonding urea-urethane end-capped supramolecular polymer. Soft Matter 2015;11:5799-803.

[33] Hiremath JG, Khamar NS, Palavalli SG, Rudani CG, Aitha R, Mura P. Paclitaxel loaded carrier based biodegradable polymeric implants: Preparation and in vitro characterization. Saudi Pharm J: SPJ 2013;21:85-91.

[34] Zhao Z, Li Y, Zhang Y. Preparation and characterization of paclitaxel loaded SF/PLLAPEG-PLLA nanoparticles via solution-enhanced dispersion by supercritical CO2. J Nanomater.

[35] Ma JH, Guo C, Tang YL, Liu HZ. ${ }^{1} \mathrm{H}$ NMR spectroscopic investigations on the micellization and gelation of PEO-PPO-PEO block copolymers in aqueous solutions. Langmuir 2007;23:9596605 . 
[36] Ma JH, Guo C, Tang YL, Wang J, Zheng L, Liang XF, et al. Salt-induced micellization of a triblock copolymer in aqueous solution: A ${ }^{1} \mathrm{H}$ nuclear magnetic resonance spectroscopy study. Langmuir 2007;23:3075-83.

[37] Kim BJ, Im SS, Oh SG. Investigation on the solubilization locus of Aniline-HCl salt in SDS micelles with ${ }^{1}$ H NMR spectroscopy. Langmuir 2001;17:565-6.

[38] Hammouda B. SANS from pluronic P85 in d-water. Eur Polym J 2010;46:2275-81.

[39] Hackley VA, Stoimenov PK, Ho DL, Sung LP, Klabunde KJ. Structure development in aerogel-processed nanocrystalline alkaline earth oxides as revealed by SANS. J Appl Crystallogr 2005;38:619-31.

[40] Cheon Lee S, Kim C, Chan Kwon I, Chung H, Young Jeong S. Polymeric micelles of poly(2ethyl-2-oxazoline)-block-poly( $\varepsilon$-caprolactone) copolymer as a carrier for paclitaxel. J Control Release 2003;89:437-46.

[41] Alexander S, Cosgrove T, Castle TC, Grillo I, Prescott SW. Effect of temperature, cosolvent, and added drug on Pluronic-flurbiprofen micellization. J Mater Chem B 2012;116:11545-51.

[42] Basak R, Bandyopadhyay R. Encapsulation of hydrophobic drugs in Pluronic F127 micelles: Effects of drug hydrophobicity, solution temperature, and pH. Langmuir 2013;29:4350-6.

[43] Sharma PK, Bhatia SR. Effect of anti-inflammatories on pluronic ${ }^{\circledR}$ F127: Micellar assembly, gelation and partitioning. Int J Pharm 2004;278:361-77.

[44] Bhattacharjee J, Verma G, Aswal VK, Hassan PA. Small angle neutron scattering study of doxorubicin-surfactant complexes encapsulated in block copolymer micelles. Pramana 2008;71:991-5.

[45] Bhattacharjee J, Verma G, Aswal VK, Patravale V, Hassan PA. Microstructure, drug binding and cytotoxicity of Pluronic P123-aerosol OT mixed micelles. RSC Advances 2013;3:23080-9.

[46] Franklin J, Jinu G, Supriya VV, Mrinal S, Hassan PA, Aswal VK, et al. Enhanced efficacy of Pluronic copolymer micelle encapsulated SCR7 against cancer cell proliferation. Macromol Biosci 2015;15:521-34.

[47] Dehvari K, Lin KS. Synthesis, characterization and potential applications of multifunctional PEO-PPO-PEO- magnetic drug delivery system Curr Med Chem 2012;19:5199-204.

[48] Lin Y, Alexandridis P. Temperature-dependent adsorption of Pluronic F127 block copolymers onto carbon black particles dispersed in aqueous media. J Mater Chem B 2002;106:10834-44. 

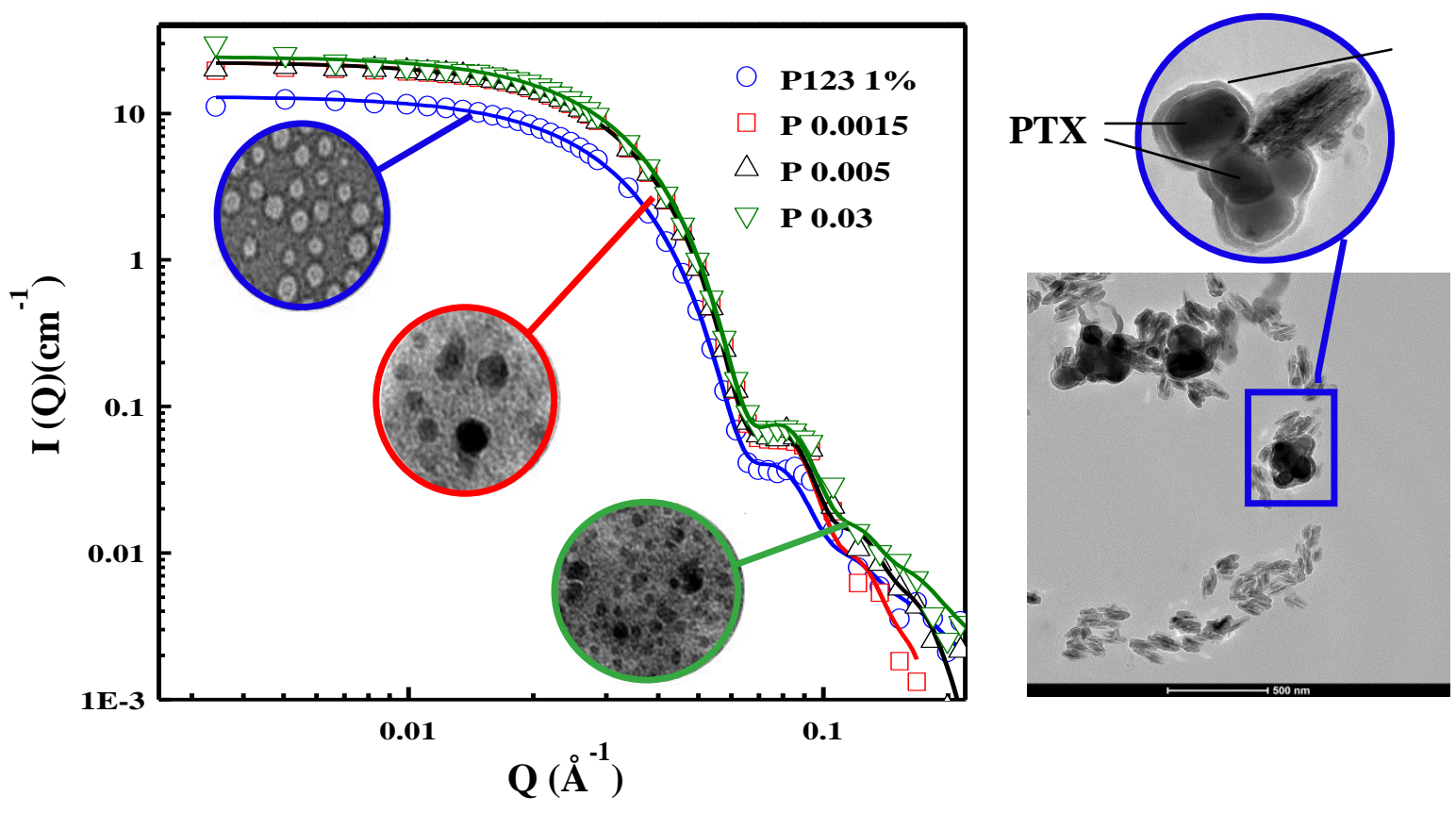

Graphical abstract 